equivocally, as scientists do: climate is not weather, and although all extreme weather events are now subject to human influence, global warming driven by greenhouse gases cannot be said to 'cause' any specific manifestation of weather in a simple deterministic sense.

Is that response enough? The question, after all, seems fair, given the dire warnings of worsening weather that are offered to the public as reasons to care about global warming. It may irritate some scientists, but in fact the question can be seen as a vindication of their efforts to spread the message that the climate problem is a clear and present danger. Most people associate the climate with the weather that they experience, even if they aren't supposed to. And they are right to wonder how and why that experience can, on occasion, leave their homes in pieces.

Given the growing interest, it is a good sign that scientists plan to launch a coordinated effort to quickly and routinely assess the extent to which extreme weather events should be attributed to climate change (see page 148). The ambitious idea is in the early stages, and its feasibility is yet to be demonstrated. It will require funding, access to climate data from around the world and considerable computer time. Funding agencies and climate centres must provide the necessary support.

As operational climate-attribution systems develop, it is important that they do not remain purely an academic exercise. To reach out to the public, attribution scientists could do worse than to ally themselves with meteorologists - including commercial providers of weather forecasts - to explain how climate change affects the risk of extreme weather. There is, after all, a lot of scope for the makers and presenters of daily weather reports to inform their listeners and viewers more solidly about consequences of climate change than they have chosen to do in the past.

Climate scientists, too, have an obligation to provide more coherent

answers to queries (or doubts) as to how global warming influences our weather. An attribution system with ample resources, running in near real time, could prevent scientists' answers to those questions seeming either too cautious or too alarmist and speculative. It could also prevent the public from getting the (false) impression that climate

"Mostpeople associate the climate with the weather that they experience, even if they aren't supposed to." research is confined to the virtual world of climate models and has little to offer when it comes to current reality, or that climate science is a quasi-experimental field that yields scary but mostly unverifiable results. The service's broad integration into people's daily lives, through the old and new mass media, would be a good way to seed greater acceptance of climate scientists' actual services to society and the problem of climate change.

There are constraints here. Attribution is only as good as the models and statistics that power it - and the various existing climate models project different trends in future extremes in some regions. There is a lack, or poor availability, of long-term observational records, and of climate data with high spatial and temporal resolution. And however it develops, climate attribution will remain rooted in probabilities. Not even the most thorough study can work out with absolute confidence the exact fingerprint of global warming in a given weather event.

What about Irene, then? A concerted attribution effort should help to resolve, in the not-so-distant future, the ongoing controversy over the effect of climate change on hurricane formation. Whatever the result, if the exercise can prevent people from building houses along the most vulnerable coastlines, it will be worth the effort.

\section{Brain burdens}

\section{Europe's shocking statistics on neurological and mental disorders demand a shift in priorities.}

$\mathrm{R}$ esearch to combat diseases, one would think, would be funded in proportion to the burden inflicted on the population. The reality is very different - witness the disparity between the huge burden caused by diseases in the developing world and the scant resources for research to tackle them.

Another disparity exists, and it is universal: the significant burden of mental and neurological illnesses of the human brain compared with the small proportion of research funds available to understand and treat them. Unlike cardiovascular disease and cancer, in most cases the burdens of brain disorders tend to manifest themselves in disabilities and in effects on the lives of the people afflicted and their carers, rather than in early deaths. That makes the footprint of these conditions harder to quantify.

So it is particularly valuable that a group of brain specialists and statisticians has produced a new quantification of the burden of brain disorders across 30 European countries. Building on a previous study, they assessed more disorders, analysed the literature and consulted national experts to validate emerging perspectives. The result? A conservative estimate that, in a typical year, about 165 million people $-38 \%$ of the total population of these countries - will have a fully developed mental illness (H. U. Wittchen et al. Eur. Neuropsychopharmacol. 21, 655-679; 2011).

The shocking statistics don't end with prevalence. A good measure of disease burden is the disability-adjusted life year (DALY) - the person-years lost in a population owing to disability and shortened life. The authors establish brain disorders - both mental and neurological - as the greatest health burden on the population, comprising $23.4 \%$ of all DALYs among men and $30.1 \%$ for women.
Many more men than women have alcohol-use disorders, especially in Eastern Europe; for both dementia and unipolar depression, the ratios of women to men are around two to one. The reasons for these latter gender differences are not understood, although depression among women seems to arise especially during their child-bearing years. The estimated number of people affected by major depression in the 30 countries studied is 30 million - the single greatest burden of all human diseases.

One piece of good news that emerges from the study is that the prevalence of individual conditions, alcoholism apart, has not grown in the past five years. The truly bad news is that only one in two people with a mental condition has ever received any professional attention, and that only $10 \%$ receive "notionally adequate" treatment — and then only after much delay following initial contact with health professionals.

What does this mean for research? The message for funding is clear: priorities need to shift. In financial terms, European research on brain diseases is much less-well supported than research on cancer, information technology, agriculture and other areas (see go.nature.com/hr2jqp). The state of the science is such that it requires major investment in all aspects, from fundamental neuroscience to psychological therapies.

There are subtler messages too. Research should target not only those brain diseases in which the prevalence in ageing populations will inevitably increase, such as Alzheimer's, but also the brains of young people, both healthy and ill. Many mental disorders emerge or begin to develop in the first two decades of life. Our knowledge of the healthy adolescent brain - a stage of still-active neural development - is rudimentary. The tantalizing prospect of therapeutic or preventive psychological or pharmaceutical interventions at such ages needs to be pursued.

This will give rise to ethical quandaries - probabilistic labels of future psychiatric disorders and over-reliance on drugs are problematic. But understanding how neural circuits develop in the young, $\rightarrow$ NATURE.COM To comment online, click on Editorials at: go.nature.com/xhunqv and how environmental and innate influences combine to disrupt them, is one of the most fascinating and difficult scientific challenges of our time. The new study re-emphasizes just how urgent it is. 